\title{
Sunset jets observed on comet 67P/Churyumov-Gerasimenko sustained by subsurface thermal lag
}

\author{
X. Shi ${ }^{1}$, X. Hu ${ }^{1}$, H. Sierks ${ }^{1}$, C. Güttler ${ }^{1}$, M. A'Hearn ${ }^{2}$, J. Blum ${ }^{3}$, M. R. El-Maarry ${ }^{4}$, E. Kührt ${ }^{5}$, S. Mottola ${ }^{5}$, M. Pajola ${ }^{6}$, \\ N. Oklay ${ }^{1}$, S. Fornasier ${ }^{7}$, C. Tubiana ${ }^{1}$, H.U. Keller ${ }^{3}$, J.-B. Vincent ${ }^{1}$, D. Bodewits ${ }^{2}$, S. Höfner ${ }^{1}$, Z.-Y. Lin ${ }^{8}$, A. Gicquel ${ }^{1}$, \\ M. Hofmann ${ }^{1}$, C. Barbieri ${ }^{9}$, P. L. Lamy ${ }^{10}$, R. Rodrigo ${ }^{11,12}$, D. Koschny ${ }^{13}$, H. Rickman ${ }^{14}$, M. A. Barucci ${ }^{7}$, \\ J.-L. Bertaux ${ }^{15}$, I. Bertini ${ }^{6}$, G. Cremonese ${ }^{16}$, V. Da Deppo ${ }^{17}$, B. Davidsson ${ }^{18}$, S. Debei ${ }^{19}$, M. De Cecco ${ }^{20}$, M. Fulle ${ }^{21}$, \\ O. Groussin ${ }^{10}$, P. J. Gutiérrez ${ }^{22}$, S. F. Hviid ${ }^{5}$, W.-H. Ip ${ }^{8}$, L. Jorda ${ }^{10}$, J. Knollenberg ${ }^{5}$, G. Kovacs ${ }^{1}$, J.-R. Kramm ${ }^{1}$, \\ M. Küppers ${ }^{23}$, L. M. Lara ${ }^{22}$, M. Lazzarin ${ }^{9}$, J. J. Lopez-Moreno ${ }^{22}$, F. Marzari ${ }^{9}$, G. Naletto ${ }^{24,6,17}$, and N. Thomas ${ }^{4}$
}

(Affiliations can be found after the references)

Received 5 August 2015 / Accepted 5 December 2015

\begin{abstract}
We present observations of sunset jets on comet 67P/Churyumov-Gerasimenko by Rosetta/OSIRIS camera. In late April 2015, when the comet was at a heliocentric distance of $\sim 1.8 \mathrm{AU}$, clusters of dust jets that originated in the Ma' at region on the comet's small lobe were identified from multiple images and were apparently sustained for about an hour beyond local sunset. Emanating from the shadowed nucleus, these jets became visible by solar illumination at their apparent sources up to only a few tens of meters above the nucleus surface. We investigate the plausibility of these jets as having been triggered by water ice sublimation and sustained by thermal lag in the subsurface beyond sunset. A general thermo-physical model was parameterized such that the thermal lag in the subsurface is consistent with the elapsed time of observation after sunset. It is found that the sublimation of water ice from a depth of $6 \mathrm{~mm}$ and with a low thermal inertia of $50 \mathrm{~W} \mathrm{~m}^{-2} \mathrm{~K}^{-1} \mathrm{~s}^{1 / 2}$ could explain the spatial pattern and evolution of the apparent sources, particularly their disappearance due to the eventual cooling of the subsurface. Our analysis suggests that these sunset jets were essentially day-side dust activities that continued after sunset. Specific observational conditions for the sunset jets constrain their possible sources to mostly within the less abrupt, dusty terrains. The uneven distribution of these jets is possibly related to subsurface inhomogeneities in the dusty area.
\end{abstract}

Key words. comets: individual: 67P/Churyumov-Gerasimenko

\section{Introduction}

Dust activities from the night side of a comet have been observed in several previous comet missions (Sekanina et al. 2004; Farnham et al. 2007, 2013; Feaga et al. 2007; Belton et al. 2008; A'Hearn et al. 2011; Bruck Syal et al. 2013). Many of these activities manifest themselves as filaments, or jets, originating in unilluminated areas on the nucleus. Some of these dust activities that occur far into the night side are classified as outbursts, probably involving super volatiles (Feaga et al. 2007; Belton et al. 2008). Those observed near sunrise are possibly triggered by sublimation of water ice and of super volatiles that have refrozen and accumulated on the surface during the night (Feaga et al. 2007; Prialnik et al. 2008). Others, and in particular jets near the dusk terminator, may be due to sublimation of water ice sustained by subsurface thermal lag (Farnham et al. 2007, 2013).

The characterization of night-side activity on comet $67 \mathrm{P} /$ Churyumov-Gerasimenko (hereafter referred to as 67P) is among the scientific objectives of the Optical, Spectroscopic, and Infrared Remote Imaging System (OSIRIS) on board the European Space Agency's Rosetta spacecraft (Keller et al. 2007). The day-side dust jets on the comet were distinguishable in OSIRIS images starting from July 2014 onwards (Lara et al. 2015). The activity from any given location follows insolation (Keller et al. 2015) and the active locations appear to be morphology-dependent (Vincent et al. 2016). As 67P is approaching perihelion, jets have been observed to emerge from the dark side of the nucleus. However, one prominent jet observed by OSIRIS before dawn was probably a mini-outburst (Knollenberg et al. 2015). More often, these night-side jets were observed close to the dusk terminator. Hereafter, we refer to such jets observable briefly after sunset as sunset jets.

In the Ma'at region on the nucleus's small lobe (Fig. 1, see also Thomas et al. (2015b) and El-Maarry et al. (2015) for definitions of all geomorphological regions on 67P mentioned in this work), sunset jets were observed as early as February 2015 in OSIRIS images. Since then, they have been more frequently and widely observed under favorable illumination conditions and viewing geometry.

\section{Observation}

Clusters of sunset jets were observed by the OSIRIS Narrow Angle Camera (NAC) near the southern border of Ma'at with Bastet on 22, 25, and 27 April 2015, when the comet was at a heliocentric distance of $\sim 1.8 \mathrm{AU}$, approaching its southernspring equinox toward perihelion (Figs. 2 and 3). The subsolar latitude in the body-fixed frame of the comet (Preusker et al. 2015) moved from $6.5^{\circ}$ to $4.7^{\circ}$ during these six days. The source area is roughly between $5^{\circ}$ to $20^{\circ}$ in longitude and $5^{\circ}$ to $30^{\circ}$ in latitude on the small lobe of the nucleus (Fig. 1). The faint dust jets are clearly distinguishable when accentuated in contrast to the dark background (Figs. 2d-f; 3d and e). Emanating from the shadowed nucleus, a sunset jet becomes illuminated at a certain point above the surface (Farnham et al. 2007), defined 


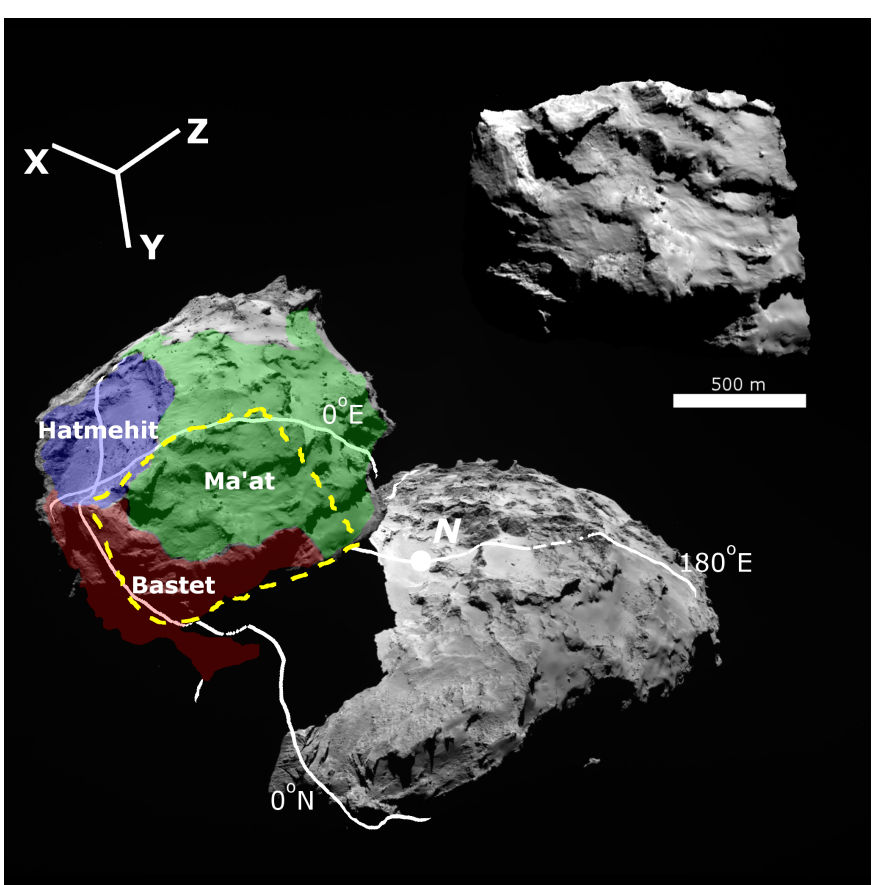

Fig. 1. Location of the source area of sunset jets in this study as outlined by dashed yellow line on the nucleus of 67P. The areas in green, red, and blue correspond to the geomorphological regions of Ma'at, Bastet, and Hatmehit, respectively, on the small lobe of the nucleus. The equator, $0^{\circ}$ and $180^{\circ}$ longitudes, and the North Pole are indicated according to the Cheops reference frame of 67P (Preusker et al. 2015). Upper right: an enlarged view of the source area.

here as the "apparent source", and eventually either fades away or becomes obscure against the illuminated background. We note that the "apparent source" of a sunset jet is distinct from its real source on the surface. Many of these jets appear linear and collimated with typical widths ranging from a few up to dozens of pixels that, when observed at a distance of $100 \mathrm{~km}$, correspond to a few up to a hundread metres. Note that the three images of Fig. $2 \mathrm{~d}-\mathrm{f}$ are in reverse order in terms of local time, with Fig. $2 \mathrm{f}$ being the earliest observation, and Fig. 2e and d taken roughly 10 and 25 min later than Fig. 2f.

An additional sequence of three images on April 25 shows the apparent evolution of jets over time around sunset (Fig. 3). Two jets are identified along the terminator immediately after dark (Fig. 3g). More jets were observed 40 min later when the area was mostly in shadow (Fig. 3h), most of which appear in two prominent clusters. It is possible that they were present but indistinguishable in the previous image, while the source area was still largely illuminated. These jets seem to have faded significantly after another $40 \mathrm{~min}$ (Fig. 3i). The fading of jets may be due to changing illumination conditions, i.e., jets were still present but almost entirely shadowed by the nucleus. Alternatively, it may indicate a weakening, i.e., a diurnal pattern, of dust activity, likely associated with water ice sublimation in the shallow subsurface.

\section{Data analysis}

The source and orientation of a linear jet can usually be determined via the method of triangulation that requires at least two successive observations under suitable illumination condition and with different viewing geometries (Sekanina et al. 2004; Farnham et al. 2013; Bruck Syal et al. 2013). The long separation in time of observations in Fig. 2 and the changing illumination condition for observations in Fig. 3 both hinder the identification of common jets from the respective sequences. However, in the case of jets emerging from an unilluminated area, the viewing geometry and shadowing effect fully determine the position of their apparent sources and constitute special constraints on the location of their sources on the nucleus.

\subsection{Improvement on viewing geometry estimation}

The viewing geometry, i.e., the position and orientation of the camera with respect to the body-fixed frame of 67P, is obtained from the SPICE kernels (Acton 1996) in the form of a translation vector,

$\hat{t}=t+\delta t$,

and a rotation matrix,

$\hat{\boldsymbol{R}}=\boldsymbol{R}+\boldsymbol{\delta} \boldsymbol{R}$,

with small errors, $\delta \boldsymbol{t}$ and $\boldsymbol{\delta} \boldsymbol{R}$, relative to the respective true (but unknown) quantities, $\boldsymbol{t}$ and $\boldsymbol{R}$. The pixel coordinates, $X, Y$, of a certain feature in the image can be determined from its coordinates in the body-fixed frame for given viewing geometry.

In this study, the viewing geometry derived from SPICE is refined when a synthetic image based on the initial estimates, $\hat{\boldsymbol{t}}$ and $\hat{\boldsymbol{R}}$, is notably misaligned from the real image. Specifically, we select at least 6 landmarks on the polyhedral shape model of $67 \mathrm{P}$ consisting of more than 16 million facets with a resolution of $\sim 2 \mathrm{~m}$ (Preusker et al. 2015), and measure the discrepancy of their synthetic pixel coordinates from those in the actual image, $\delta X, \delta Y$. In case of large discrepancies not accountable by the uncertainty of the shape model, typically $\sim 1$ pixel, $\delta \hat{\boldsymbol{t}}$ and $\delta \hat{\boldsymbol{R}}$ are estimated from $\delta X, \delta Y$ in the least-squares sense and subsequently corrected from $\hat{\boldsymbol{t}}$ and $\hat{\boldsymbol{R}}$ (note that the overhead symbol, “»", applies to $\delta \hat{\boldsymbol{t}}$ and $\delta \hat{\boldsymbol{R}}$, which are estimates themselves and, thus, contain errors). This is an iterative process until $\delta X$, $\delta Y$ are minimized and converge to $\sim 1$ pixel on average, suggesting an improvement to the initial viewing geometry given by SPICE kernels.

\subsection{Determination of apparent source of jet}

Figure 4 sketches the geometrical condition for the observation of a sunset jet. Because the position of the Sun in the comet's body-fixed frame is known, the position of the apparent source, which is at a certain distance above the surface, is the intersection of the camera line-of-sight (LOS) of the apparent source with the Sun-apparent-source line. The apparent source is identified as the peak-intensity pixel along the jet profile. With known focal length of the camera, the LOS of the apparent source is derived from its pixel coordinates. We inspect the visibility of the Sun along the LOS at an interval of $1 \mathrm{~m}$, starting from the intersection point of the LOS with the shape model outward. Then, the three-dimensional coordinates of the apparent source are found as those of the first illuminated point on the LOS.

We apply a reduced-resolution global shape model with $\sim 200000$ facets (Preusker et al. 2015) for our subsequent analysis and illustration. The reasons are two-fold: firstly, the resolution of the shape model of $\sim 15 \mathrm{~m}$ roughly corresponds to the typical widths of $\sim 10 \mathrm{~m}$ for the jets in the observations and, hence, assimilates inevitable errors in our geometric analysis; secondly, a regional shape model with the same resolution will be adopted for our subsequent thermo-physical analysis for computational efficiency. 

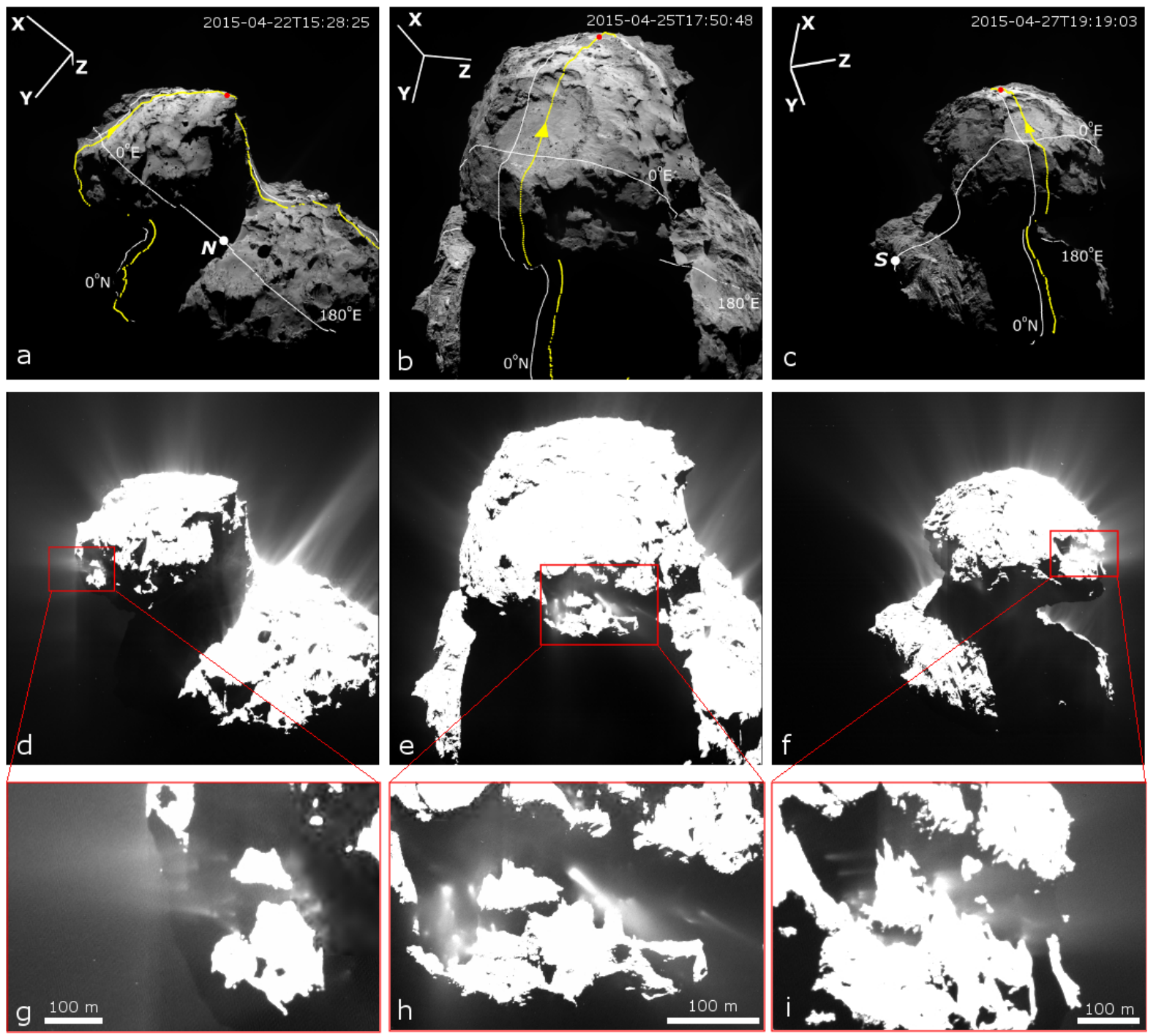

Fig. 2. OSIRIS observations of sunset jets in Ma'at. a)-c) Nucleus of 67P observed at UTC 2015-04-22 15:28:25, 2015-04-25 17:50:48, and 2015-04-27 19:19:03, respectively. The dotted line in yellow indicates the solar ground track during the corresponding rotation cycle, and the red dot indicates the subsolar point at the time of observation. The direction of motion of the subsolar point on the nucleus is indicated by the yellow arrow. The subsolar longitude in the body-fixed frame is $310^{\circ}, 315^{\circ}$, and $323^{\circ}$ for the three observations, respectively. The annotated axes, the $0^{\circ}$ and $180^{\circ}$ longitudes, the equator, and the North/South Poles are as indicated in Fig. 1. d)-f) Contrast-stretched views of a)-c), revealing dust jets against dark background. g)-i) Enlarged views of the region outlined by red rectangles in d)-f), showing individually distinguishable sunset jets as well as their source area. (Detailed information of all images used in this work is given in Table 1).

As an example, Fig. 5a shows the apparent sources of sunset jets observed in Fig. $2 \mathrm{~h}$, marked as green dots. In this case, all apparent sources are located at some distance of less than $40 \mathrm{~m}$ above the nucleus surface (see Fig. $5 \mathrm{~d}$ for a perspective view). It is worth noting that one apparent source (No. 8) rises by less than $2 \mathrm{~m}$. The fact that all apparent sources are close to the surface results from the mild undulations of topography in the area and, in particular, the long shadow cast by the uplift in the west shortly after sunset (see Fig. $5 \mathrm{c}$ and d). The determined apparent sources for all observed jets in Figs. 2 and 3 have an average distance of $25 \mathrm{~m}$, with a maximum of $80 \mathrm{~m}$, from the ground. The apparent sources clearly ascend as time progresses beyond sunset.

\subsection{Derivation of possible jet sources on the nucleus}

A jet plane can be specified by the LOSs of two points along a jet. The first point is conveniently chosen as the apparent source; the second point is along the apparent direction of a jet, i.e., the observed minimum gradient from the contours of jet intensity (dotted blue lines in Fig. 5a). It may be difficult to distinguish such a direction when a jet appears too diffuse or indistinguishable from other jets; in those cases, only the apparent sources are determined. The intersection of the jet plane and the nucleus indicates a trail of possible sources of a jet on the nucleus surface, extending backwards from the intersection of the LOS of the apparent source with the nucleus (Fig. 5b). To further narrow down the possible sources, the following constraints are taken into account:

- A jet should not be obstructed by local topography between its source and the apparent source.

- A jet should not be illuminated between its source and the apparent source.

The second condition not only recapitulates that the jet source is unilluminated, but also excludes the possibility of partial illumination of the jet before the apparent source. 
Table 1. Information on OSIRIS images used in this work.

\begin{tabular}{|c|c|c|c|c|c|}
\hline Figure & Image ID & Imaging time (UTC) & $\begin{array}{c}r_{\mathrm{h}} \\
(\mathrm{AU})^{*}\end{array}$ & $\begin{array}{c}d \\
(\mathrm{~km})^{* *}\end{array}$ & $\begin{array}{c}\text { Pixel } \\
\text { resolution }(\mathrm{m})\end{array}$ \\
\hline 1 & WAC_2014-09-21T12.49.58.768Z_ID30_1397549900_F17 & 2014-09-21 12:51:08 & 3.33 & 27.6 & 2.56 \\
\hline $2 \mathrm{a}, \mathrm{d}, \mathrm{g}$ & NAC_2015-04-22T15.27.09.343Z_ID30_1397549000_F22 & $2015-04-22 \quad 15: 28: 25$ & 1.80 & 145.6 & 2.67 \\
\hline $2 \mathrm{~b}, \mathrm{e}, \mathrm{h}$ & NAC_2015-04-25T17.49.32.608Z_ID30_1397549001_F22 & $2015-04-25$ 17:50:48 & 1.78 & 92.9 & 1.69 \\
\hline $2 \mathrm{c}, \mathrm{f}, \mathrm{i}$ & NAC_2015-04-27T19.17.47.350Z_ID30_1397549000_F22 & 2015-04-27 19:19:03 & 1.76 & 138.8 & 2.54 \\
\hline $3 \mathrm{a}, \mathrm{d}, \mathrm{g}$ & NAC_2015-04-25T05.05.51.540Z_ID30_1397549001_F22 & 2015-04-25 05:07:07 & 1.78 & 91.5 & 1.66 \\
\hline $3 \mathrm{~b}, \mathrm{e}, \mathrm{h}$ & NAC_2015-04-25T05.45.51.563Z_ID30_1397549001_F22 & 2015-04-25 05:47:07 & 1.78 & 91.5 & 1.66 \\
\hline $3 \mathrm{c}, \mathrm{f}, \mathrm{i}$ & NAC_2015-04-25T06.25.51.520Z_ID30_1397549001_F22 & 2015-04-25 06:27:07 & 1.78 & 91.3 & 1.66 \\
\hline $7,13 \mathrm{c}$ & NAC_2014-09-19T23.09.00.386Z_ID30_1397549100_F22 & 2014-09-19 23:10:10 & 3.33 & 28.1 & 0.48 \\
\hline $11 \mathrm{a}, \mathrm{b}$ & NAC_2014-10-22T00.23.03.311Z_ID30_1397549800_F22 & 2014-10-22 00:24:14 & 3.13 & 10.1 & 0.15 \\
\hline $11 \mathrm{c}$ & NAC_2014-10-19T12.22.15.525Z_ID30_1397549600_F22 & 2014-10-19 12:23:26 & 3.15 & 10.0 & 0.15 \\
\hline $12 \mathrm{a}, \mathrm{b}, \mathrm{c}$ & NAC_2015-03-28T16.12.49.393Z_ID30_1397549000_F82 & 2015-03-28 16:14:04 & 1.99 & 20.0 & 0.33 \\
\hline $13 \mathrm{a}, \mathrm{b}$ & NAC_2015-03-28T18.06.47.592Z_ID30_1397549700_F22 & 2015-03-28 18:08:03 & 1.99 & 26.4 & 0.45 \\
\hline
\end{tabular}

Notes. ${ }^{(*)}$ Heliocentric distance. ${ }^{(*)}$ Distance between the spacecraft and the center of the nucleus.
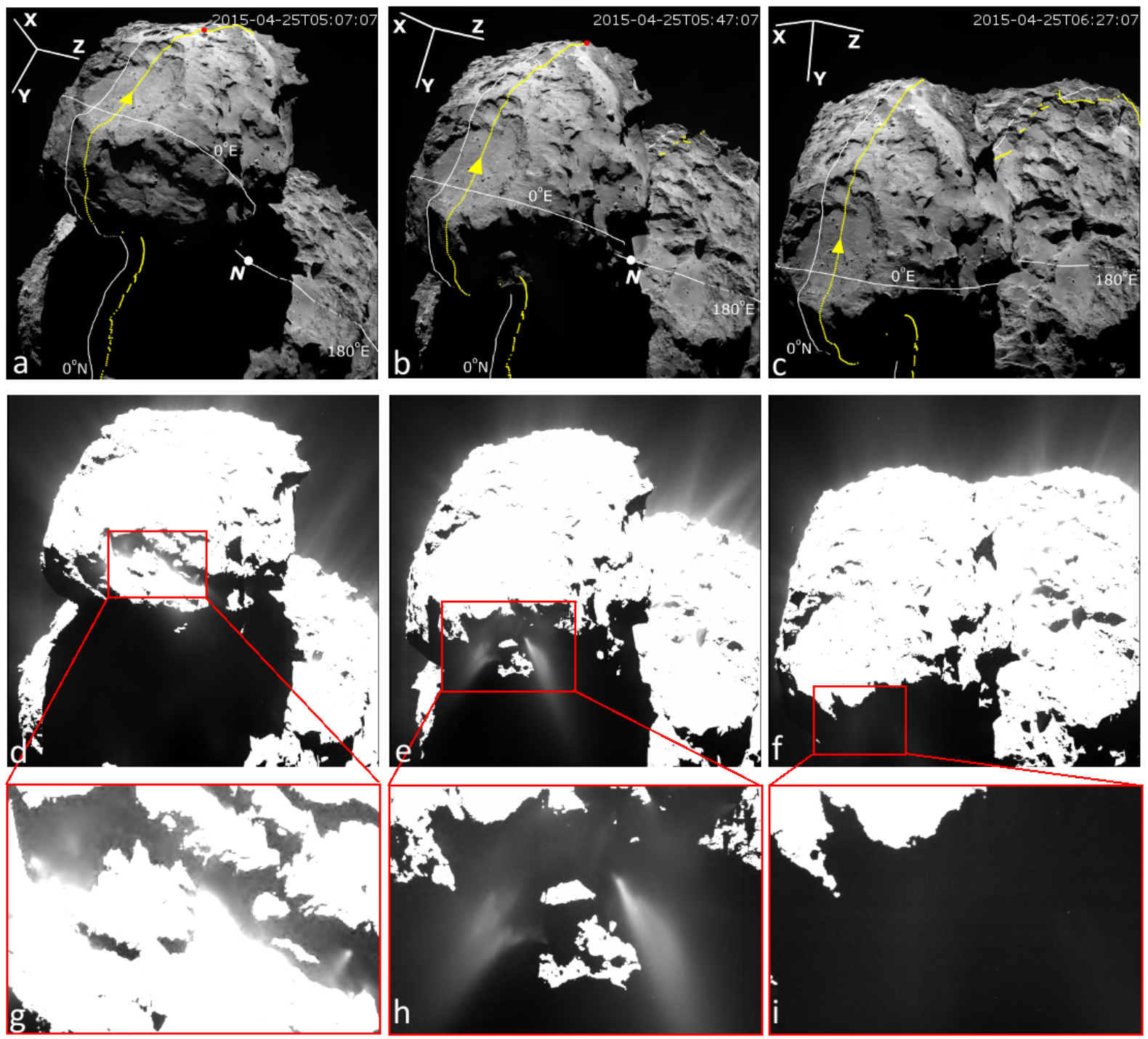

Fig. 3. OSIRIS observations of sunset jets in Ma'at taken approximately one rotation cycle earlier than the observation in Fig. 2b. a)-c) NAC images taken every $40 \mathrm{~min}$ from UTC 2015-04-25 05:07:07, showing the sunset process over the source area as the nucleus rotates around its $z$ axis. The annotations are the same as in Fig. 2a-c. In c), the subsolar point is shielded by the small lobe of the nucleus and thus not visible. d)-f) Contrast-stretched views of a)-c), showing the evolution of sunset jets as the source area gradually enters shadow. g)-i) Enlarged views of the region outlined by red rectangles in $\mathbf{d})-\mathbf{f}$ ). 


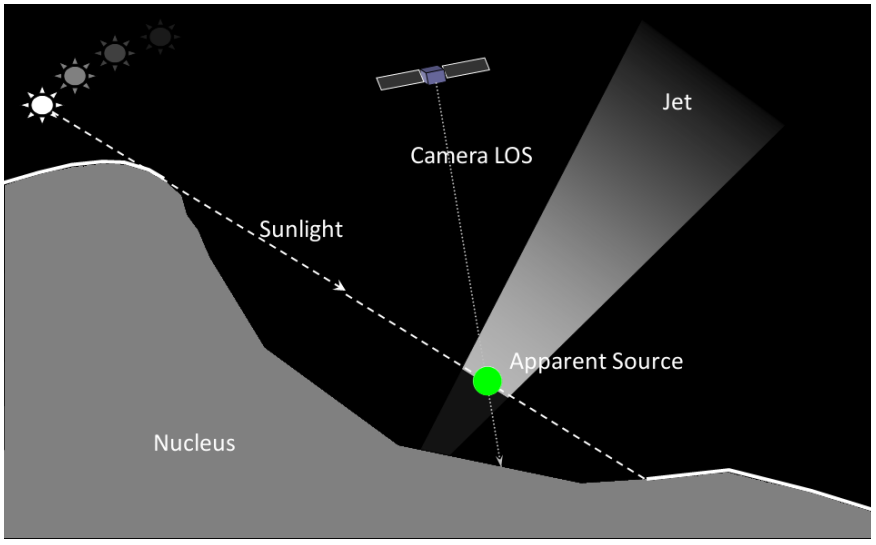

Fig. 4. Schematic illustration of the observational geometry for sunset jets.

As illustrated in Fig. 5b, we discretize the trail into possible source points $2 \mathrm{~m}$ apart, and reconstruct the jet by connecting each point with the apparent source. The validity of the above two conditions is then tested for each reconstruction; those points at which either condition is not satisfied are ruled out as possible sources. For the observation in Fig. 5a, eight jet planes are identified, and the resulting trails of possible jet sources are shown in Fig. $5 \mathrm{c}$ and d. Of course, in the original viewing geometry of Fig. 5a, the trails appear collinear with the apparent direction of the jets (Fig. 5c). At least three trails (No. 4, 6, and 8) are significantly shortened by the imposed constraints, limiting the sources of jets to the vicinity of the apparent sources.

We identified jet planes and derived possible jet sources for all three observations with distinct sunset jets (Fig. 2g-i). It is found that, along the trails of possible sources, the jet plane has a large angle of inclination with respect to the local topography (Fig. 6), defined as the angle between the surface normal of the jet plane and that of the corresponding facet of the shape model. In more than $80 \%$ of the cases, the jet planes are nearly perpendicular to the nucleus surface, i.e., with an inclination between $80^{\circ}$ to $100^{\circ}$. No plane is found with an inclination smaller than $50^{\circ}$ or greater than $130^{\circ}$. These statistics, based on three separate observations with different viewing geometry, suggest that the observed jets (assumed to be linear, of course) cannot be randomly oriented with respect to the nucleus surface. They rarely propagate in an oblique direction from the nucleus but are, in general, nearly perpendicular to the surface.

In Fig. 7, the derived possible sources for the observed jets in Fig. 2 are superposed on a NAC image of the area with good illumination condition. The width of the trails roughly corresponds to the horizontal uncertainty of $\sim 15 \mathrm{~m}$ in the derived source locations. With only a few exceptions, these trails of possible sources rarely traverse but rather halt by sloped topography (relative to the smooth, presumably flat areas), which again results from the highly localized illumination conditions for the sunset jets. Parts of the trails are accentuated where a jet would be inclined from the nucleus surface by from $60^{\circ}$ up to $120^{\circ}$, i.e., roughly perpendicular to the surface (opaque portions of the trails in Fig. 7). Hence, they are more likely to contain the actual sources of jets than the rest (transparent portions) of the trails. These possible source locations are close to their apparent sources, which are at some short distance above the nucleus. Thus, the sources of these sunset jets are probably located dominantly in the area with distinct dust deposits that are prevalent in Ma'at (Thomas et al. 2015b; El-Maarry et al. 2015). It is also possible that some jets originated from common areas, as indicated by the intersections of trails.

\section{A model for thermal lag and dust activity}

The thermal lag in the subsurface sustaining the sunset jets is, arguably, the most intuitive scenario with water ice as the dominant volatile species (Farnham et al. 2013). Jets may also be caused by the sublimation of super-volatiles, such as $\mathrm{CO}$ or $\mathrm{CO}_{2}$, more likely governed by the interior condition (Prialnik \& Bar-Nun 1990; Enzian 1999; Belton et al. 2007; Belton 2010). The plausibility of the two scenarios can be tested via a modeling approach (Prialnik et al. 2004; Gortsas et al. 2011). The first scenario will be specifically investigated below, given that the sunset jets in our study are consistently observed near the illuminated area and rarely far into the night side and, hence, suggest a possible diurnal pattern of activity.

We apply a generic thermo-physical model for the subsurface of the nucleus, with a dry dust layer overlying dust-ice mixtures (Keller et al. 2015). The nucleus is assumed to be homogeneous, such that the subsurface temperature, $T$, varies according to the one-dimensional heat equation,

$c \rho \frac{\partial T}{\partial t}=\frac{\partial}{\partial z}\left(\kappa \frac{\partial T}{\partial z}\right)$

with $t, z$ indicating time and depth, respectively. $\rho$ is the mass density, and $c, \kappa$ denote the heat capacity and effective conductivity of the material, respectively.

On the surface of the nucleus we assume that the absorbed energy flux due to solar radiation, $Q_{\odot}$, and "self-heating" of the nucleus, i.e., absorption of thermal emission from other areas, $Q_{\mathrm{IR}}$, is balanced by local thermal emission and heat conduction (Lagerros 1997; Gutiérrez et al. 2001; Ivanova \& Shulman 2006; Davidsson \& Rickman 2014; Keller et al. 2015),

$Q_{\odot}+Q_{\mathrm{IR}}=\left.\left(\sigma \epsilon T^{4}-\kappa_{\mathrm{D}} \frac{\partial T}{\partial z}\right)\right|_{z=0^{+}}$,

where $\sigma$ is the Stefan-Boltzmann constant, and $\epsilon$ the emissivity of the surface. $\kappa_{\mathrm{D}}$ denotes the heat conductivity of the dust layer. At the bottom of the dust layer, or ice front, $z=Z$, energy is consumed for sublimation of volatiles, i.e. (Kührt \& Keller 1994),

$-\left.\kappa_{\mathrm{D}} \frac{\partial T}{\partial z}\right|_{z=Z^{-}}=-\left.\kappa_{\mathrm{I}} \frac{\partial T}{\partial z}\right|_{z=Z^{+}}+\ell F$,

where $\kappa_{\mathrm{I}}$ is the heat conductivity of the dust-ice mixture. $\ell$ is the latent heat of the volatile species. The mass flux of outgassing material through the dust layer, $F$, is given by (Gundlach et al. 2011),

$F=\Psi \alpha P_{\mathrm{V}} \sqrt{\frac{m}{2 \pi k_{\mathrm{B}} T_{Z}}}\left(\mathrm{~kg} \mathrm{~m}^{-2} \mathrm{~s}^{-1}\right)$,

in the Knudsen regime, and the heat transport via gas diffusion is neglected. $k_{\mathrm{B}}$ is the Boltzmann constant and $m$ the molecular mass of the volatile gas. $T_{Z}$ is the sublimation temperature. $P_{\mathrm{V}}$ is the vapor pressure, which for pure water ice is given by (Gundlach et al. 2011),

$P_{\mathrm{V}}=3.23 \times 10^{12} \exp \left(-6134.6 / T_{Z}\right)(\mathrm{Pa})$.

The sublimation coefficient for pure water ice is obtained experimentally as (Gundlach et al. 2011),

$\alpha=0.146+\frac{0.854}{1+\exp \left(57.78-11580 / T_{Z}\right)}$. 
a

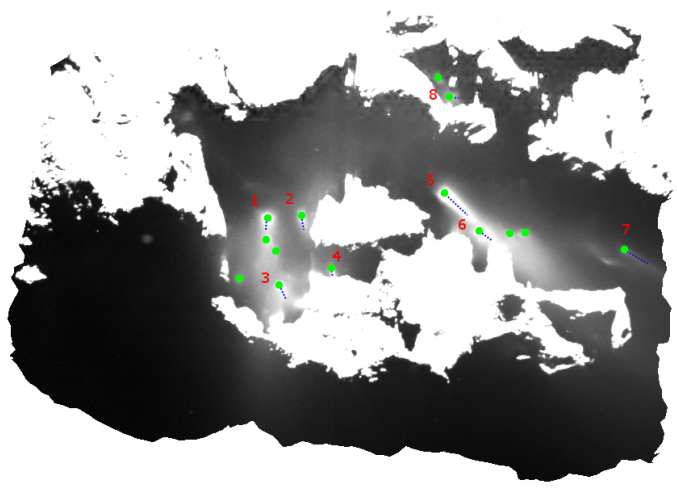

C

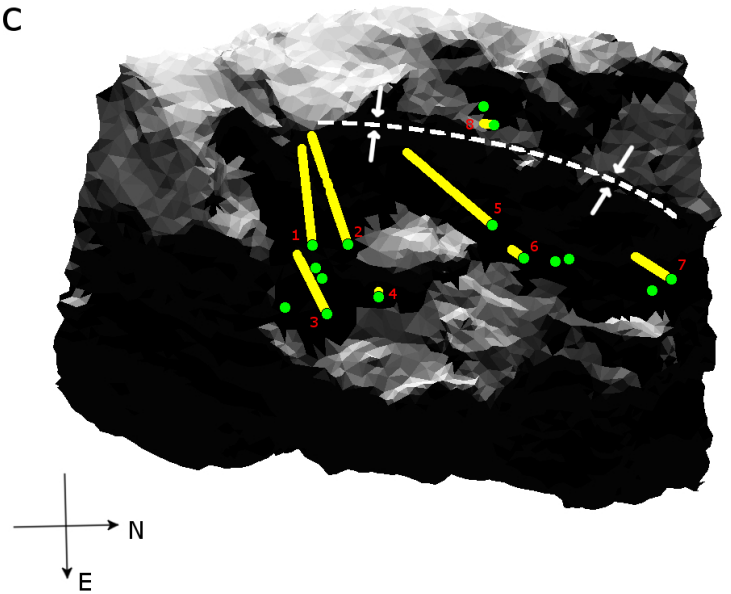

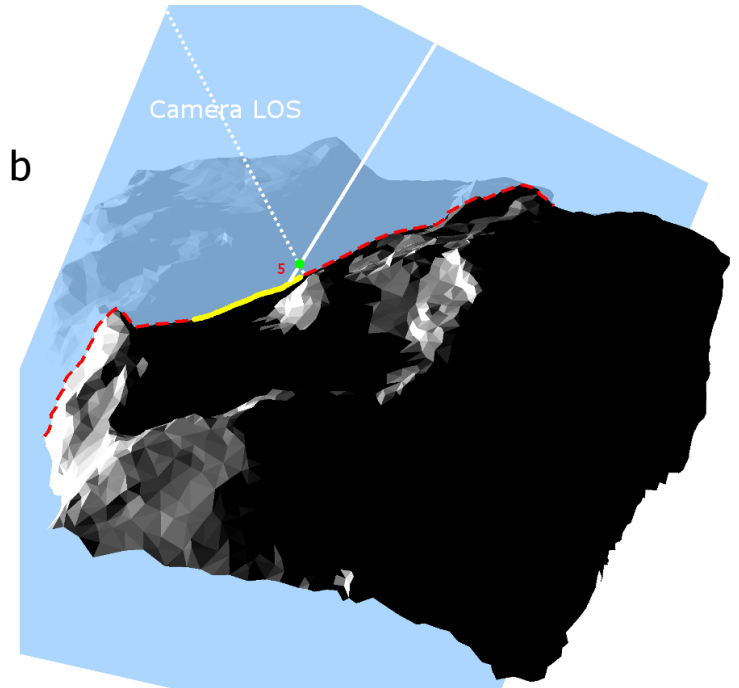

d

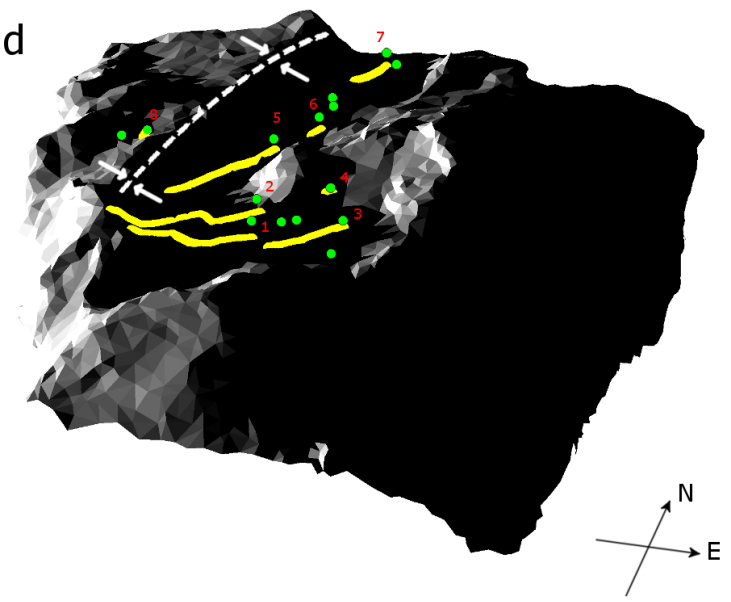

Fig. 5. Apparent sources and possible sources of sunset jets on the nucleus. a) Annotated observation of sunset jets as in Fig. 2h. The apparent sources of jets are marked by green dots. Jets with distinguishable directions are indicated by the dotted blue line and are numbered. b) Oblique view of the source area based on the shape model for illustrating the connections between the apparent source (the green dot), the jet plane (translucent blue plane), and possible sources on the nucleus (the yellow trail) for jet No. 5 in a). The dashed red line indicates the intersection of the jet plane with the nucleus. The solid white line indicates a possible orientation of the jet as reconstructed by connecting the apparent source and a possible source on the surface. c) Determined apparent sources and trails of possible sources on the nucleus of all observed jets in a) with the same viewing geometry. The dashed white arc and the arrows indicate the uplift to the west of the source area. d) Same as c), but with the same viewing geometry as b) to better illustrate the elevation of the apparent sources from the ground as well as the uplift to the west.

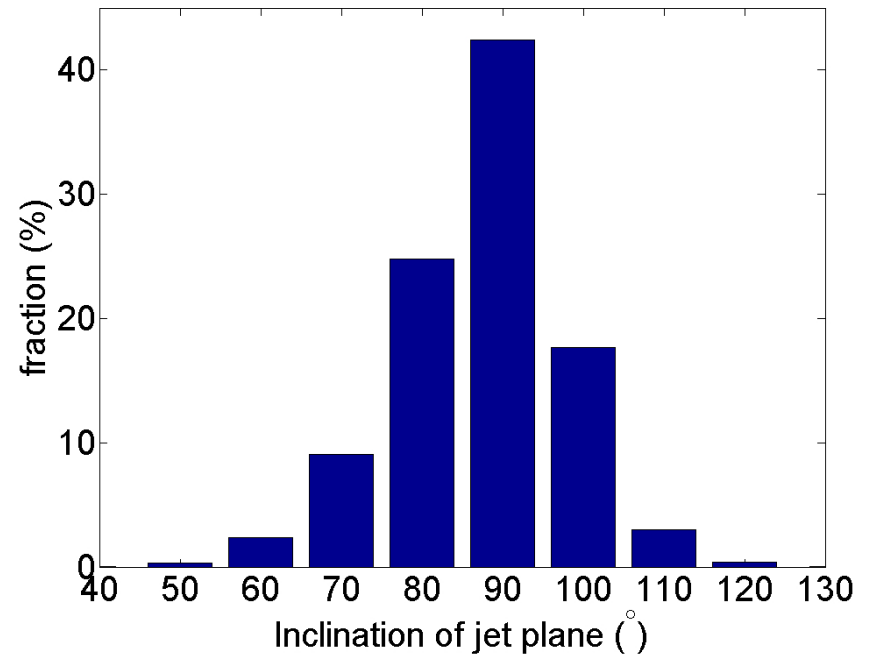

Fig. 6. Statistics of inclination of the jet planes with respect to the shape model along the trails of possible jet sources derived from all observations in Fig. 2.
See also Kossacki \& Markiewicz (2013) for a relevant discussion. The dust layer, assumed to be homogeneous, suppresses the uprising gas flux by a factor (Gundlach et al. 2011),

$\Psi=\frac{1}{1+h / 7.31}$

where the height of dust layer, $h=Z / d_{\mathrm{P}}$, is measured in units of diameters of the constituting particles, $d_{\mathrm{P}}$.

We further impose, as the lower boundary condition, that (Kührt 1984; Davidsson \& Skorov 2002),

$\left.\frac{\partial T}{\partial z}\right|_{S}=0$,

i.e., the temperature gradient vanishes at a certain depth, $S$.

\subsection{Model parameters}

The particle size, $d_{\mathrm{P}}$, is a critical modeling parameter, as it affects not only heat transfer but also tensile strength of the dust layer 


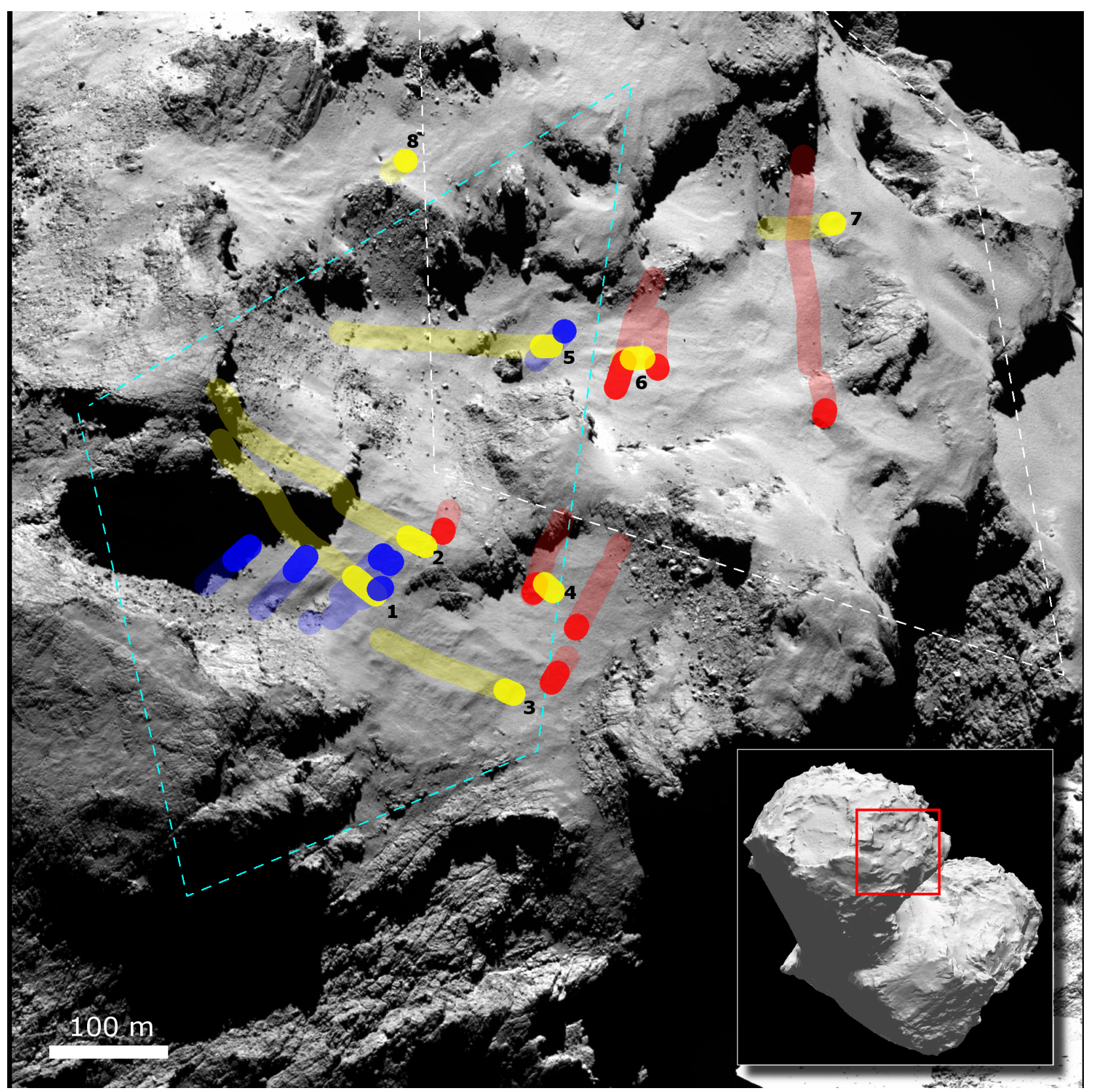

Fig. 7. Trails of possible sources of sunset jets superposed on a context image. Trails in red, yellow, and blue, correspond to jets identified in Fig. $2 \mathrm{~g}, \mathrm{~h}$, and i, respectively. The opaque parts of the trails indicate possible sources with reconstructed jets that are inclined by $60^{\circ}$ to $120^{\circ}$ relative to the local surface; jets originating from the transparent parts of the trails would be inclined by less than $60^{\circ}$ or more than $120^{\circ}$ with respect to the local surface. The background image was taken by OSIRIS NAC at UTC 2014-09-19 23:10:10. The field of view with respect to the nucleus (shape model as in Preusker et al. 2015) is indicated by the red rectangle in the lower right panel. The dashed line in light-blue and white roughly outline the areas in Figs. 11 and 12, respectively.

(Skorov \& Blum 2012; Blum et al. 2014). Dust particles (in the form of aggregates) in the coma of 67P vary in size (Schulz et al. 2015; Rotundi et al. 2015; Fulle et al. 2015). Determination of the particle size distribution of the dust layer is indeed a challenging task. Here, we adopt $d_{\mathrm{P}}=1 \mathrm{~mm}$ for an idealized, uniform dust layer as a preliminary attempt.

We assume $c=1000 \mathrm{~J} \mathrm{~K}^{-1} \mathrm{~kg}^{-1}$ and $\rho=500 \mathrm{~kg} \mathrm{~m}^{-3}$, the latter of which is consistent with the determined bulk density of 67P (Sierks et al. 2015). The surface bolometric Bond albedo is 0.05 and $\epsilon=1$. We use $\kappa=\kappa_{\mathrm{D}}=\kappa_{\mathrm{I}}=$ $0.005 \mathrm{~W} \mathrm{~K}^{-1} \mathrm{~m}^{-1}$. Note that this corresponds to a thermal inertia, $\sqrt{\kappa \rho c}=50 \mathrm{~W} \mathrm{~m}^{-2} \mathrm{~K}^{-1} \mathrm{~s}^{1 / 2}$, the upper limit by August 2014 as reported by Gulkis et al. (2015). This value of $\kappa$ roughly accomodates the increasing contribution of radiative heat transfer in the dust layer, approximated as (Gundlach \& Blum 2012),

$\kappa^{\mathrm{rad}} \approx 3.6 \sigma T^{3} \frac{1-\phi}{\phi} d_{\mathrm{P}}\left(\mathrm{W} \mathrm{K}^{-1} \mathrm{~m}^{-1}\right)$,

where $\phi$ is the volume filling factor of the dust layer. Assuming $\phi=0.3, \kappa^{\mathrm{rad}}$ ranges between 0.002 and $0.01 \mathrm{~W} \mathrm{~K}^{-1} \mathrm{~m}^{-1}$ for $T \in$ $[150,300] \mathrm{K}$.

The observed duration of these jets into the night side yields some constraints on modeling parameters. The timescale of thermal conduction is defined by (Huebner et al. 2006),

$\tau_{\kappa}=c \rho Z^{2} / \kappa(\mathrm{s})$. 


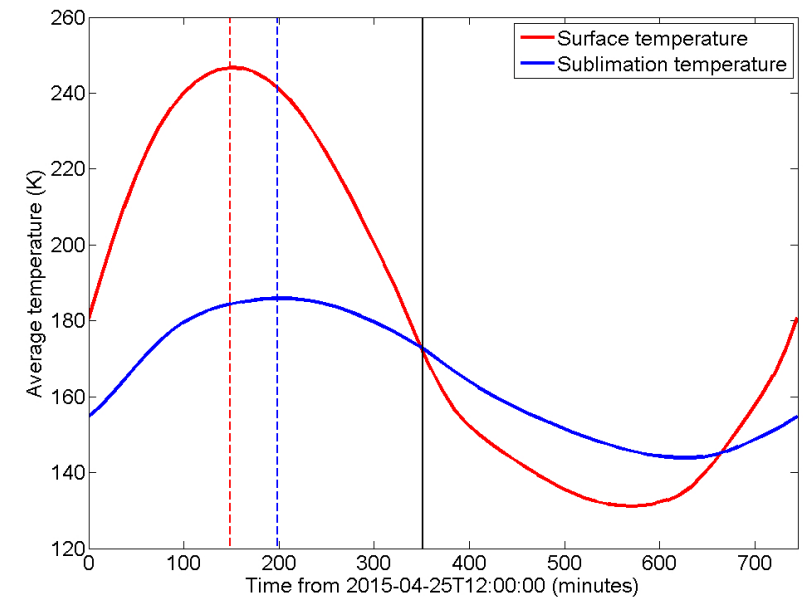

Fig. 8. Diurnal variation of the surface and sublimation temperatures averaged over the entire source area during one rotational period on April 25, 2015. The solid black line indicates the time of observation for Fig. 2b. The dotted red and blue lines indicate the location of maximum values in average surface and sublimation temperature, respectively; the offset is a measure of thermal lag in the subsurface layer.

Since jets were observed beyond $40 \mathrm{~min}$ after sunset, we adopt $Z=6 \mathrm{~mm}$, resulting in a comparable timescale of $\sim 1 \mathrm{~h}$. This value is also compatible with results from VIRTIS, the Visible and Infrared Thermal Imaging Spectrometer onboard Rosetta, indicating the overall depletion of water ice in the uppermost $1 \mathrm{~mm}$ layer (Capaccioni et al. 2015). We note that the dust layer overlies the diurnal skin depth for $67 \mathrm{P}$ at $\sqrt{t_{\mathrm{R}} \kappa / \pi \rho c} \approx 1 \mathrm{~cm}$, with $t_{\mathrm{R}}(\mathrm{s})$ being the rotation period of the comet. It must be stressed that the dust layer in the model is much thinner than, as it differs from, the observed dust cover, possibly several meters thick in the Ma'at region (Thomas et al. 2015b). The thickness of the dust layer, $Z$, is a generic model parameter characterizing the depth of icy dust with no consideration of morphology.

It is assumed that a constant interior temperature of $50 \mathrm{~K}$ is reached below the depth of $S=20 \mathrm{~m}$.

\subsection{Modeled surface and sublimation temperature}

We apply this model to the area showing sunset jets and investigate subsurface thermal conditions of activity for all six observations in Figs. 2 and 3. The topography is represented by a $\sim 10000$-facet local shape model truncated from the original 200 000-facet global shape model (Preusker et al. 2015). Using the boundary conditions set by Eqs. (4), (5), and (10), we solve Eq. (3) numerically for temperature, $T(t, z)$, at discrete depths for each facet of the shape model via the Crank-Nicolson method. In the iterative process, we search for a "numerical" diurnal skin depth $z_{\mathrm{N}}$, where the temperature fluctuates by less than $1 \mathrm{~K}$ during one rotation period; this is typically at a few diurnal skin depths. The solution is considered to be stable once the temperature above the numerical diurnal skin depth converges to that after one rotation period, i.e., $T\left(t, z \leq z_{\mathrm{N}}\right) \cong T\left(t+t_{\mathrm{R}}, z \leq z_{\mathrm{N}}\right)$. In so doing, the choice of interior temperature and the corresponding depth, $S$, has negligible impact on the solution.

The diurnal variation of the modeled surface and sublimation temperatures averaged over the area during one rotation period on April 25 are shown in Fig. 8. The significant attenuation of the variation of sublimation temperature compared with that of the surface temperature results from: 1) the low thermal inertia of the dust layer that thwarts the heating and cooling of the subsurface; and 2) the consumption of energy by sublimation in the subsurface. The thermal lag in the subsurface, as approximated by the analytic value of $\tau_{\kappa}$, is indicated by the delay of $\sim 50 \mathrm{~min}$ in the maximum sublimation temperature relative to the maximum surface temperature. Figure 9 shows the surface and sublimation temperatures in the source area at the time of observation for Fig. $2 \mathrm{~h}$ with the same viewing geometry. The surface temperature follows instantly insolation, and ranges between $130 \mathrm{~K}$ in the shadow (excluding areas that are always in shadow during the rotation cycle) and $290 \mathrm{~K}$ in the illuminated area (Fig. 9a). The sublimation temperature, $T_{Z}$, varies less notably between $145 \mathrm{~K}$ and $193 \mathrm{~K}$ (Fig. 9b). To accentuate the pattern of the sublimation temperature in the shadowed area, we use a rescaled colormap in Fig. 9c and d, accordingly, and mask the illuminated areas by white facets. In most of the shadowed area, the subsurface retains higher temperatures than the surface that has cooled down more rapidly after sunset. In particular, we note that the maximum $T_{Z}$ in the shadowed area always verges on the dusk terminator (Fig. 9d), and the temperature gradually decreases further away into the night side. This accentuates that the modeled $T_{Z}$ is insolation-driven and sustained by thermal lag.

We define the footprint of apparent source for a jet as the closest point on the surface. Since jet planes intersect the nucleus at some large angles always above $60^{\circ}$ and, along a majority of the intersection, close to $90^{\circ}$ (Fig. 6), these footprints approximate, if not coincide with, the actual sources of jets on the nucleus. We note that the surface temperature at the footprints of apparent sources has decreased significantly and is consistently below $170 \mathrm{~K}$ (Fig. 9c). On the other hand, the footprints are associated dominantly with high sublimation temperatures of above $180 \mathrm{~K}$ close to the dusk terminator (Fig. 9d).

\subsection{Terminator of inactivity for dust jets}

We consider two criteria for dust activity from the nucleus (Blum et al. 2014; Gundlach et al. 2015). Firstly, the gas pressure below (or inside) the dust layer should exceed the tensile strengths of the constituting particles (Kührt and Keller 1994; Möhlmann 1995). The tensile strength for a layer of loose dust deposits can be predicted as,

$P_{\text {tensile }}=2.6 \phi_{\mathrm{P}} d_{\mathrm{P}}^{-2 / 3}(\mathrm{~Pa})$,

with $\phi_{\mathrm{P}}$ as the volume filling factor of aggregates and the particle size, $d_{\mathrm{P}}$, in millimeters (Skorov \& Blum 2012; Blum et al. 2014). Assuming $\phi_{\mathrm{P}}=0.5$, the minimum vapor pressure of $P_{\mathrm{V}} \approx 1 \mathrm{~Pa}$ is necessary for triggering dust activity. Hereafter, we always use the underline, "_", to denote the lower limit of a quantity explicating a necessary condition for dust activity. Secondly, the drag force exerted by the outgassing flux on dust particles should overcome gravity of the nucleus. Here, we consider a lower limit of the outgassing flux for lifting spherical particles of diameter, $d$, at rest on the nucleus against gravity (Brin 1980; Huebner et al. 2006),

$\underline{F}=\frac{d \rho g}{2 v_{\mathrm{G}}}\left(\mathrm{kg} \mathrm{m}^{-2} \mathrm{~s}^{-1}\right)$,

where $g=2 \times 10^{-4} \mathrm{~m} \mathrm{~s}^{-2}$ and $v_{\mathrm{G}}=400 \mathrm{~m} \mathrm{~s}^{-1}$ are the surface gravity acceleration of 67P (approximated as a point mass), and the thermal speed of the sublimating gas, respectively. It may be noteworthy that, in the case of sunset jets, the surface temperature may be significantly lower than the sublimation temperature, so that the outgassing flux, $F$, may decrease towards the surface as a result of condensation of gas molecules through 
a
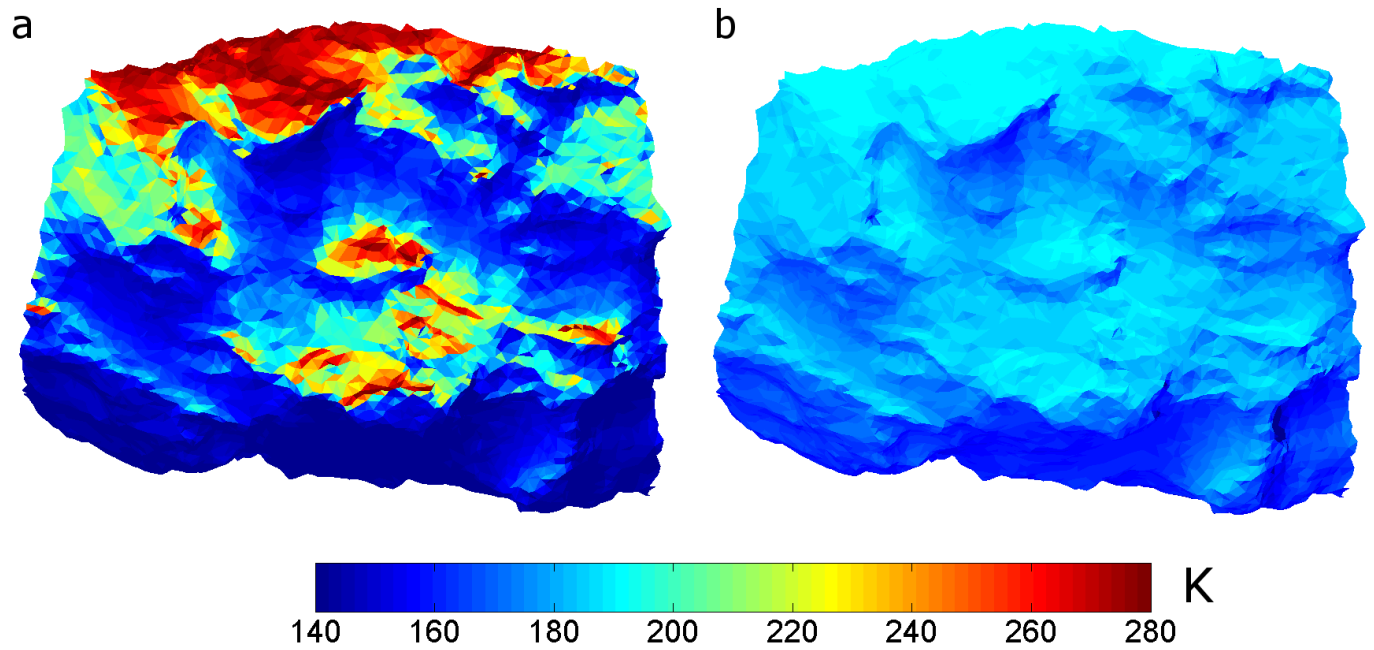

C
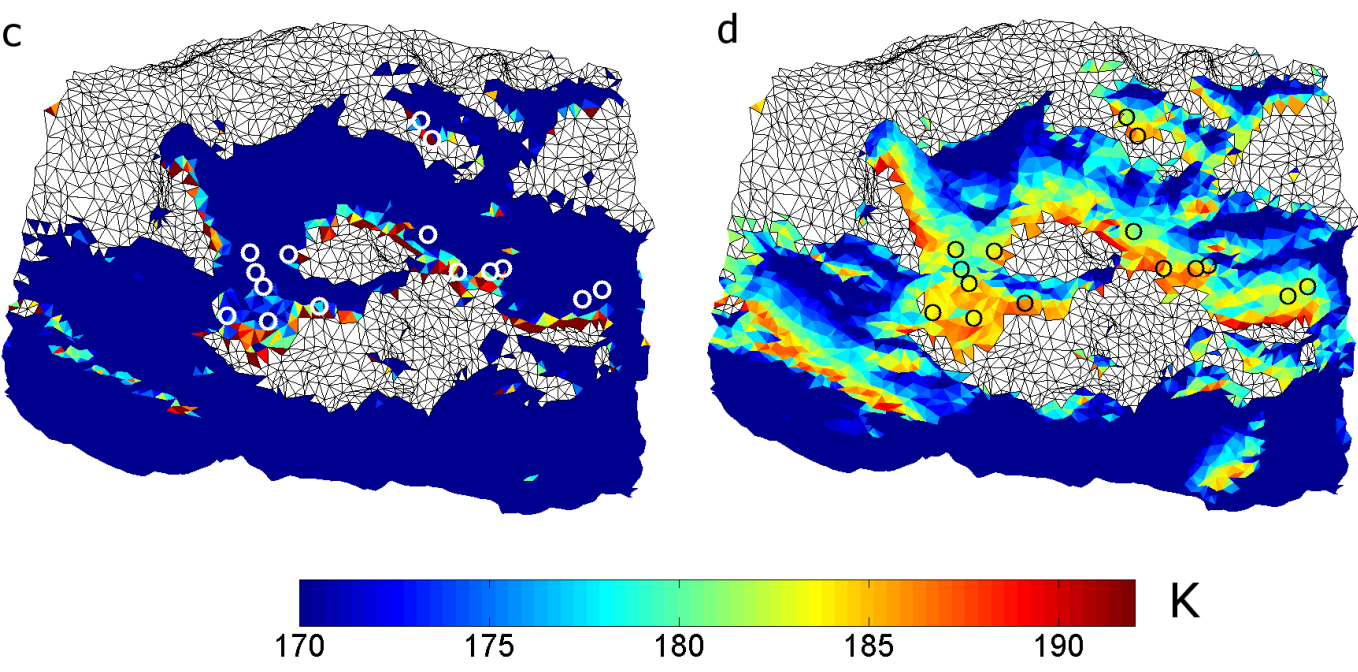

Fig. 9. Modeled temperatures in the source area at the time of observation of Fig. $2 \mathrm{~h}$ based on a local shape model. a) Surface temperature. b) Sublimation temperature. c) and d) same results as in a) and b), respectively, for the unilluminated areas. A refined colormap is used to show the variation in sublimation temperature. Facets corresponding to the illuminated areas are masked in white.

the dust layer (De Sanctis et al. 2015). Hence, it is reasonable to consider an extreme case of complete removal of the dust layer, where we assume $d=Z=6 \mathrm{~mm}\left(d \neq d_{\mathrm{p}}\right)$ in Eq. (14). In this case, a minimum outgassing flux of $\underline{F} \approx 1 \times 10^{-6} \mathrm{~kg} \mathrm{~m}^{-2} \mathrm{~s}^{-1}$ can be derived.

According to the model, the sublimation temperatures in the source area could well exceed the minimum of $T_{Z} \approx 175 \mathrm{~K}$ needed for sustaining the outgassing flux of $\bar{F} \approx 1 \times 10^{-6} \mathrm{~kg} \mathrm{~m}^{-2} \mathrm{~s}^{-1}$. However, $P_{\mathrm{V}} \approx 1 \mathrm{~Pa}$ is an order of magnitude higher than the maximum vapor pressure achieved. Additional numerical simulations with particle sizes up to $1 \mathrm{~cm}$ (still assuming a low thermal conductivity of up to $0.1 \mathrm{~W} \mathrm{~K}^{-1} \mathrm{~m}^{-1}$, to account for the timescale of conduction of $\sim 1 \mathrm{~h}$ ) suggest that water vapor pressure is unlikely to overcome the tensile strength of a homogeneous dust layer up to a few centimeters in thickness, as noted by Blum et al. (2014) and Gundlach et al. (2015). This work is not meant to address this rather profound issue in cometary physics, and certainly not to discuss a sufficient condition for dust activities. The more realistic scenario of a nonuniform dust layer composed of particles with a certain size distribution and packing structure may be worth considering (Skorov et al. 2011). In addition, the role of super-volatiles, such as $\mathrm{CO}$ and $\mathrm{CO}_{2}$, in facilitating, or driving, the observed dust activities will be investigated in future studies.

Nevertheless, it is insightful to conceptualize another terminator in addition to the dusk terminator (of illumination), defined by $T_{Z}=T_{Z}$, that separates the regions of possible activity (i.e., if we neglect tensile strengths), where $T_{Z} \geq T_{Z}$, and absolute inactivity, where $T_{Z}<T_{Z}$, for dust jets driven by water vapor. We refer to this as the terminator of inactivity (TOI). While the dusk terminator and the TOI both depend on local horizon or topography, the latter is distinct in that it reflects the effects of both insolation and thermal inertia of the overlying layer.

In all observations, the footprints of apparent sources are never found beyond the TOI or in the region of inactivity (Fig. 10). It can be inferred that the actual sources of these jets should not stray beyond the TOI, either. This suggests that the observed jets are likely day-side dust activities sustained by the slowly decreasing subsurface temperature after sunset, rather than outbursts.

As noted in Sect. 2, the subsolar latitude changed by less than $2^{\circ}$ during the six days of observations. Therefore, despite 

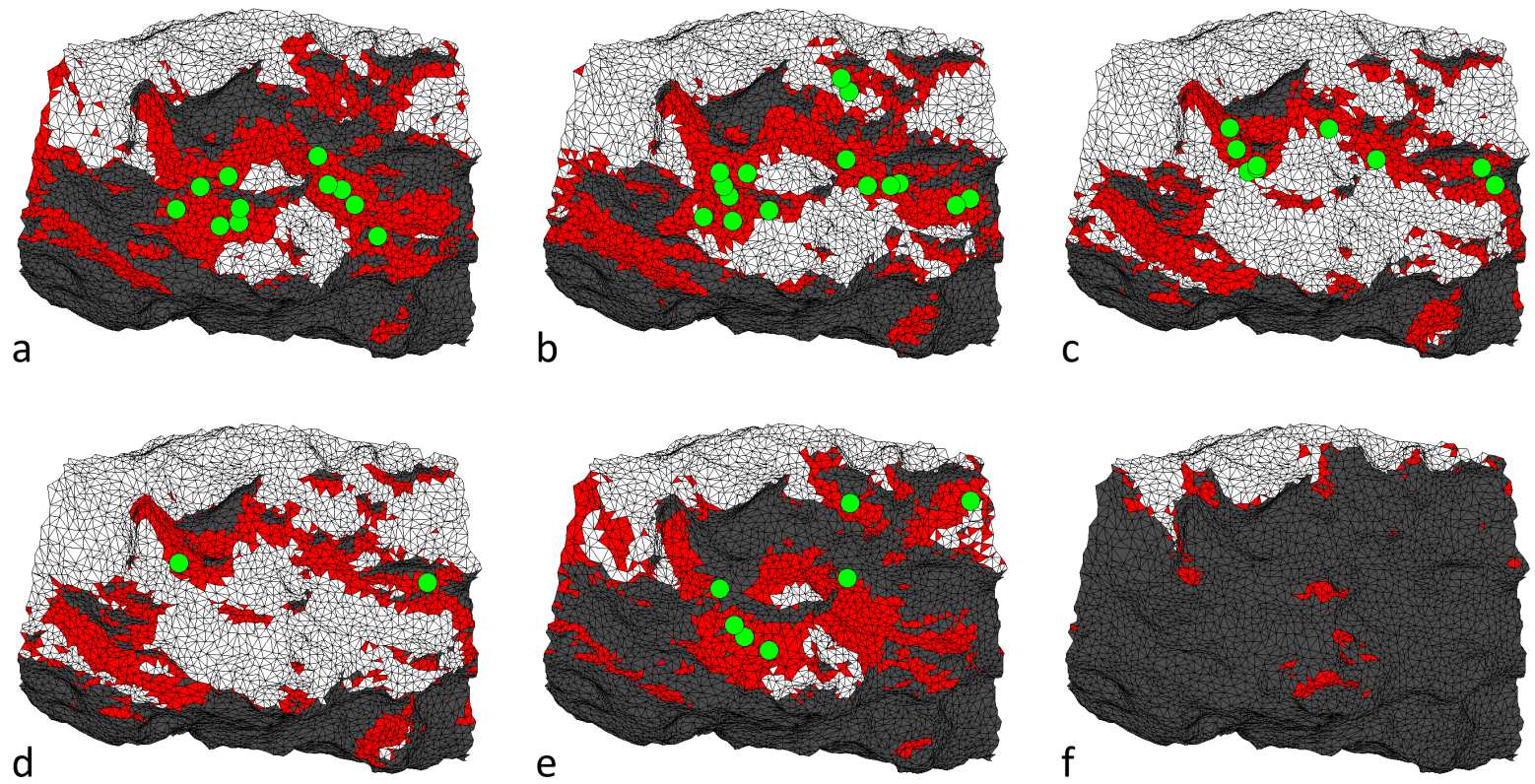

Fig. 10. Characterization of the source area based on the illumination condition and modeled sublimation temperature. The white facets correspond to the illuminated areas. Unilluminated areas with sublimation temperature above $175 \mathrm{~K}$ are represented by red facets; those with sublimation temperature lower than $175 \mathrm{~K}$ are indicated by dark gray facets. Filled green circles indicate the footprints of apparent sources of the observed sunset jets. The dusk terminator corresponds approximately to the boundary between white and red facets, while the TOI corresponds to the boundary between red and gray facets. a)-c) For observations in Fig. 2g-i. d)-f) For observations in Fig. 3g-i. No apparent sources are identified in f).

different viewing geometries, Figs. $2 \mathrm{~d}-\mathrm{f}$ and $3 \mathrm{~d}-\mathrm{f}$ in fact constitute six consecutive observations of the source area during sunset. The evolution of the dusk terminator and that of the TOI are illustrated in Fig. 10 (with panels viewed in the order of d, $c, b$, a, e, and f). The observed sunset jets, in effect, evolve in accordance with the evolution of the region of possible activity in shadow (marked as red in Fig. 10) as roughly bounded by the two terminators. When the source area is (still) broadly illuminated, only two sunset jets are distinguished that originate from the confined area in shadow near the dusk terminator and within the region of possible activity (Fig. 10d); whereas, any dust activity sourced from the illuminated area is obscured by the brightness of the sunlit surface. As the shadow expands, more sunset jets are observable and the number of apparent sources becomes significant when the region of possible activity in shadow is widespread (Fig. 10c, b, a, and e). No distinct jets can be observed by the time the region of possible activity has almost diminished (Fig. 10f). On one hand, this phenomenon can be attributed to the successive retreat of the illuminated area, rendering the dust emission beyond the dusk terminator observable above the apparent sources; on the other hand, the region of inactivity steadily expands as $T_{Z}$ declines after sunset, so that dust activity diminishes and the apparent sources eventually disappear. We note that the absence of apparent sources from the observation does not actually exclude the existence of dust activity; however, it should indicate that activity is indeed less prominent where $T_{Z}$ has further decreased as explicated by the model. Thus, the sunset jets are likely associated with a subdiurnal pattern, i.e., sustained by the thermal lag in the subsurface.

\section{Possible correlation with regional morphology}

The exact jet sources on the nucleus are unclear; however, they are more likely to stay close to the apparent sources and, thus, reside mostly in the dust-covered areas (Fig. 7). While the dust cover of the source area appears largely featureless in Fig. 7,

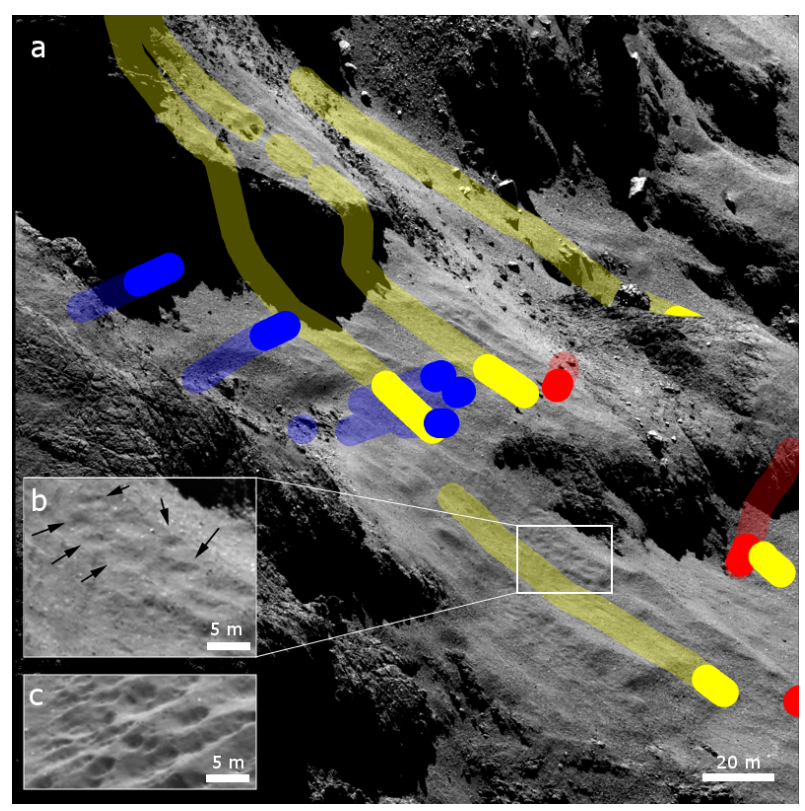

Fig. 11. Morphology of the source area of sunset jets. a) NAC image acquired on October 22, 2014, at a distance of $\sim 10 \mathrm{~km}$ with an oblique view of the source area at a higher spatial resolution than that of Fig. 7. The image covers an area roughly outlined by dashed light-blue line in Fig. 7, and the trails of possible sources of sunset jets are displayed accordingly. b) Enlarged view of the area highlighted in the white rectangle. The arrows indicate some subdued pitted texture in the dust cover. c) Cropped NAC image acquired on October 19, 2014 at a similar distance, showing the distinct pitted texture elsewhere in the Ma'at region at the same size-scale for comparison.

alternative and closer views of part of the area, with higher resolutions of $\sim 15 \mathrm{~cm} /$ pixel, reveal some visibly pitted texture of the dust-covered terrains (Fig. 11). Similar and, possibly, 

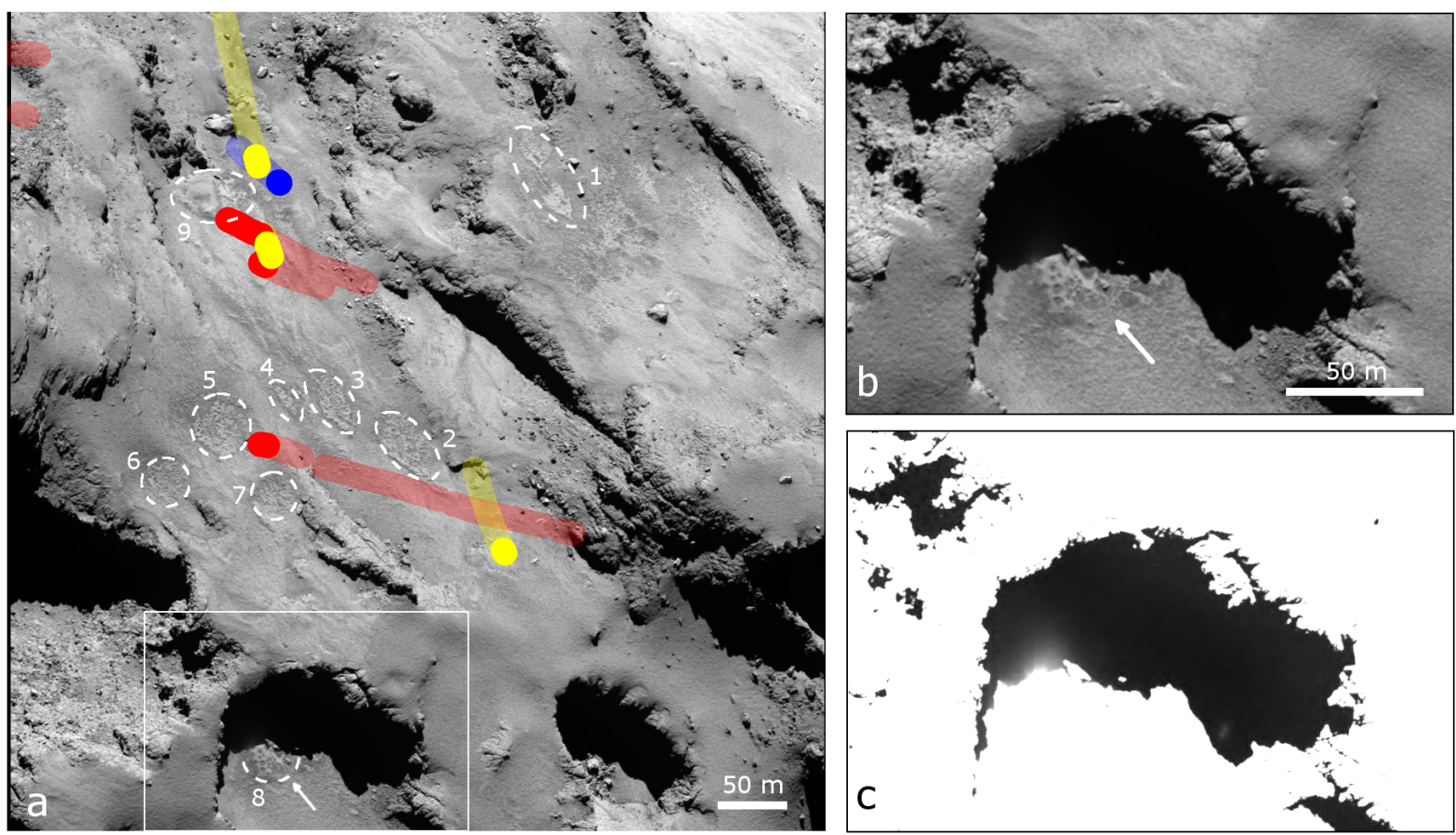

Fig. 12. a) Part of the source area imaged during the close flyby of $67 \mathrm{P}$ on March 28,2015 , at a distance of $\sim 20 \mathrm{~km}$. The image covers an area roughly outlined by the dashed white line in Fig. 7, and the trails of possible sources of sunset jets are displayed as in Fig. 7. The honeycomb features are highlighted in the dashed white circles and numbered. b) Enlarged view of the area outlined by the solid white line in a), showing one of the honeycomb structures. c) Contrast-stretched view of b) showing possible dust emission from the honeycomb feature against dark background.

more developed pitted terrains with thinning dust deposits have been noted in other areas in/near the Ma'at region (Fig. 11c, El-Maarry et al. 2015). More recent images taken during the close flyby on March 28, 2015, with resolution comparable to that of Fig. 7, reveal finer and more distinct patterns of the dusty surface (Figs. 12 and 13). Notably, various "honeycomb" features are present and may resemble ensembles of pits as shown in Fig. 11. The observations imply subsurface inhomogeneities on different scales, ranging from dozens of meters (roughly the size of a single honeycomb feature) down to submeter level (a few pixels in the image). Thomas et al. (2015a) also noted heterogeneity existing on decimeter scales or smaller in some dusty areas on 67P. On the other hand, if the uneven distribution and collimation of the sunset jets were due to inhomogeneities in structure or composition of the subsurface, the scale of such inhomogeneities would then be tens of meters, roughly comparable to the size of a honeycomb feature. Intriguingly, one of the honeycomb features, shown in Fig. 12b, appears to be an active source of dust emission at the time of observation (Fig. 12c). This may further indicate that dust activities are morphologydependent on a small scale.

We note that several honeycomb features distinct in Fig. 13 were not distinguishable in Fig. 7 taken six months earlier, when the dust cover at corresponding locations appeared far smoother. This strongly suggests that the dust cover evolves over time. It may be worth pointing out that Figs. 12 and 13 were acquired earlier than the observation of sunset jets analyzed in this study. Hence, the honeycomb features cannot be the result of the observed jet activities. Nevetheless, as discussed, sunset jets were probably common insolation-driven dust activities that continued beyond sunset due to subsurface thermal lag. Therefore, it is certainly possible that the honeycomb features resulted from nominal dust activity in the region during the time period from September 2014 through March 2015.

\section{Discussion}

OSIRIS has acquired and continues to accumulate high resolution images of night-side dust activities on 67P. Our preliminary data analysis and model results substantiated that the observed sunset jets in the Ma'at region exhibit a pattern characteristic of being sustained by thermal lag in the subsurface of one to two hours, and these jets were essentially day-side jets lasting beyond sunset to become conspicuous against the shadowed nucleus. The specific observational conditions for the sunset jets effectively constrained their possible source locations mostly within the dust-covered areas. The dusty areas, likely evolving in time, exhibit clear topographic variations reflecting possible subsurface inhomogeneities on a fine scale of tens of meters, that may contribute to the uneven distribution and intensity of the observed dust activity.

While the sunset jets were likely sustained by thermal lag in the shallow subsurface, i.e., close to the depth of water ice front, it is uncertain that they could be driven by the sublimation of only water ice. At the heliocentric distance of $\sim 1.8 \mathrm{AU}$, a homogeneous dust layer of millimeter-sized particles, even if weakly structured, should remain stable, because the tensile strengths of dust particles could not be overcome by the water vapor pressure. Note that this result, as derived from the general conclusion by Blum et al. (2014) and Gundlach et al. (2015), is not restricted to the case of sunset jets from the night side, but applies also to the day-side dust activities. On the other hand, in the unrealistic but, nonetheless, illustrative case, the outgassing flux of water vapor is well sufficient for lifting millimeter-sized dust particles against gravity of 67P. As discussed above, the conditions for dust activities are a fundamental question that is far beyond the scope of this study. A proper treatment would necessitate a much more sophisticated and elaborate modeling attempt. For instance, the strengths and permeability of an inhomogeneous dust layer and 


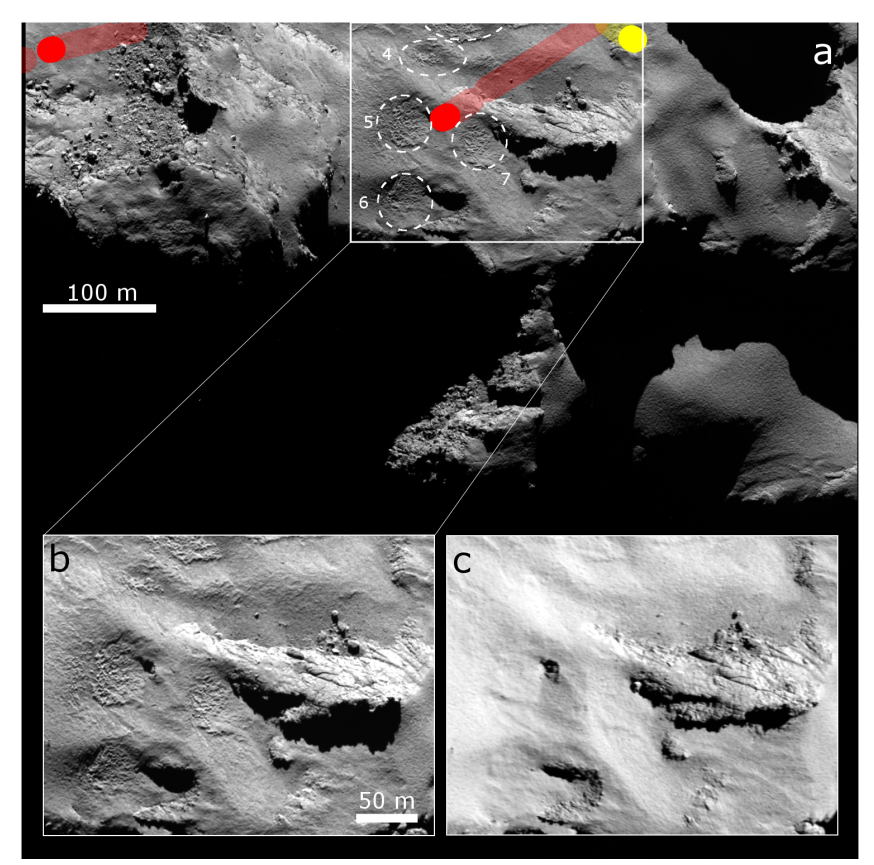

Fig. 13. a) Alternative view of the area with several honeycomb features. The honeycomb features are indicated by dashed white circles and numbered as in Fig. 12a. This image was taken roughly two hours after Fig. 12 during the flyby on March 28, 2015, at a distance of $\sim 26 \mathrm{~km}$. The trails of possible sources for observed sunset jets are indicated as well. b) Enlarged, unannotated view of the area highlighted in the white rectangle in a). c) Image cropped from Fig. 7 (taken on Septemper 19, 2014), showing the same area as in b). For better comparison, c) is coregistered to b). The dust cover appeared smoother at the corresponding locations of the honeycomb features six months earlier.

the pressure of the permeating gas flow therein may be a relevant topic of investigation. Inhomogeneities could arise from nonuniform sizes of the constituting particles as well as varying packing structures of the dust layer. This might give rise to nonuniform outgassing and, thus, enable localized dust emissions from the nucleus surface on fine spatial scales below the resolution of the images of the jets.

On the other hand, the present analysis does not take the role of super-volatiles into account, such as $\mathrm{CO}$ and $\mathrm{CO}_{2}$. Intuitively, given the temperatures derived in this work, the sublimation of $\mathrm{CO}$ and $\mathrm{CO}_{2}$ within a few diurnal skin depths would sustain strong, continuous outgassing and dust emission after sunset. However, the sublimation of $\mathrm{CO}$ or $\mathrm{CO}_{2}$ in the subsurface depends not only on insolation but also strongly on heat transport in the interior of the nucleus, and will significantly lower the surface and subsurface temperatures (Gortsas et al. 2011). Our future work will entail the application of a more comprehensive and consistent thermo-physical model to investigate the plausibility of the diurnal pattern of sublimation of super-volatiles, such as $\mathrm{CO}$ and $\mathrm{CO}_{2}$ (Hässig et al. 2015), and its relevance to the observed dust activities after sunset.

Acknowledgements. We thank an anonymous reviewer for providing many in sightful comments that significantly improved the manuscript. OSIRIS was built by a consortium led by the Max-Planck-Institut für Sonnensystemforschung, Göttingen, Germany, in collaboration with CISAS, University of Padova, Italy, the Laboratoire d'Astrophysique de Marseille, France, the Instituto de Astrofísica de Andalucia, CSIC, Granada, Spain, the Scientific Support Office of the European Space Agency, Noordwijk, The Netherlands, the Instituto Nacional de Técnica Aeroespacial, Madrid, Spain, the Universidad Politéchnica de Madrid, Spain, the Department of Physics and Astronomy of Uppsala University, Sweden, and the Institut für Datentechnik und Kommunikationsnetze der Technischen Universität Braunschweig, Germany. The support of the national funding agencies of Germany (DLR), France (CNES), Italy (ASI), Spain (MEC), Sweden (SNSB), and the ESA Technical Directorate is gratefully acknowledged.

\section{References}

A'Hearn, M. F., Belton, M. J. S., Delamere, W. A., et al. 2011, Science, 332, 1396

Acton, C. H. 1996, Planet. Space Sci., 44, 65

Belton, M. J. S. 2010, Icarus, 210, 881

Belton, M. J. S., Thomas, P., Veverka, J., et al. 2007, Icarus, 187, 332

Belton, M. J. S., Feldman, P. D., A'Hearn, M. F., et al. 2008, Icarus, 198, 189

Blum, J., Gundlach, B., Mühle, S., et al. 2014, Icarus, 235, 156

Brin, G. D. 1980, ApJ, 237, 265

Bruck Syal, M., Schultz, P. H., Sunshine, J. M., et al. 2013, Icarus, 222, 610

Capaccioni, F., Coradini, A., Filacchione, G., et al. 2015, Science, 347, 628

Davidsson, B. J. R., \& Rickman, H. 2014, Icarus, 243, 58

Davidsson, B. J. R., \& Skorov, Y. V. 2002, Icarus, 159, 239

De Sanctis, M. C., Capaccioni, F., Ciarniello, M., et al. 2015, Nature, 525, 7570

El-Maarry, M. R., Thomas, N., Giacomini, L., et al. 2015, A\&A, 583, A26

Enzian, A. 1999, Space Sci. Rev., 90, 131

Farnham, T. L., Wellnitz, D. D., Hampton, D. L., et al. 2007, Icarus, 187, 26

Farnham, T. L., Bodewits, D., Li, J.-Y., et al. 2013, Icarus, 222, 540

Feaga, L. M., A'Hearn, M. F., Sunshine, J. M., et al. 2007, Icarus, 191, 134

Fulle, M., Della Corte, V., Rotundi, A., et al. 2015, ApJ, 802, L12

Gortsas, N., Kührt, E., Motschmann, U., et al. 2011, Icarus, 212, 858

Gulkis, S., Allen, M., von Allmen, P., et al. 2015, Science, 347, 709

Gundlach, B., \& Blum, J. 2012, Icarus, 219, 618

Gundlach, B., Skorov, Y. V., \& Blum, J. 2011, Icarus, 213, 710

Gundlach, B., Blum, J., Keller, H. U., et al 2015, A\&A, 583, A12

Gutiérrez, P. J., Ortiz, J. L., Rodrigo, R., et al. 2001, A\&A, 374, 326

Hässig, M., Altwegg, K., Balsiger, H., et al. 2015, Science, 347, 276

Huebner, W. F., Benkhoff, J., Capria, M,-T., et al. 2006, Heat and Gas Diffusion in Comet Nuclei (Bern: International Space Science Institute)

Ivanova, A. V., \& Shulman, L. M. 2006, Adv. Space Res., 38, 1932

Keller, H. U., Barbieri, C., Lamy, P., et al. 2007, Space Sci. Rev., 128, 433

Keller, H. U., Mottola, S., Davidsson, B., et al. 2015, A\&A, 583, A34

Knollenberg, J., Lin, Z.-Y., Hviid, S., et al. 2015, A\&A, submitted

Kossacki, K. J., \& Markiewicz, W. J. 2013, Icarus, 224, 172

Kührt, E. 1984, Icarus, 60, 512

Kührt, E., \& Keller, H. U. 1994, Icarus, 109, 121

Lagerros, J. S. V. 1997, A\&A, 325, 1226

Lara, L. M., Lowry, S., Vincent, J.-B., et al. 2015, A\&A, 583, A9

Möhlmann, D. 1996, Planet. Space Sci., 44, 541

Preusker, F., Scholten, F., Matz, K.-D., et al. 2015, A\&A, 583, A33

Prialnik, D., \& Bar-Nun, A. 1990, ApJ, 363, 274

Prialnik, D., Benkhoff, J., \& Podolak, M. 2004, in Comets II (Tucson: University of Arizona Press), 359

Prialnik, D., A'Hearn, M. F., \& Meech, K. J. 2008, MNRAS, 388, L20

Rotundi, A., Sierks, H., Della Corte, V., et al. 2015, Science, 347, 3905

Sierks, H., Barbieri, C., Lamy, P. L., et al. 2015, Science, 347, 1044

Schulz, R., Hilchenbach, M., Langevin, Y., et al. 2015, Nature, 518, 216

Sekanina, Z., Brownlee, D. E., Economou, T. E., et al. 2004, Science, 304, 1769

Skorov, Y., \& Blum, J. 2012, Icarus, 221, 1

Skorov, Y. V., Lieshout, R. V., Blum, J., et al. 2011, Icarus, 212, 867

Thomas, N., Davidsson, B., El-Maarry, M. R., et al. 2015a, A\&A, 583, A17

Thomas, N., Sierks, H., Barbieri, C., et al. 2015b, Science, 347, 440

Vincent, J.-B., Oklay, N., Pajola, M., et al. 2016, A\&A, in press,

DOI: $10.1051 / 0004-6361 / 201527159$

1 Max-Planck-Institut für Sonnensystemforschung, Justus-vonLiebig-Weg 3, 37077 Göttingen, Germany

e-mail: shi@mps.mpg.de

2 Department of Astronomy, University of Maryland, College Park, MD 20742-2421, USA

3 Institut für Geophysik und extraterrestrische Physik (IGEP), Technische Universität Braunschweig, Mendelssohnstr. 3, 38106 Braunschweig, Germany

4 Physikalisches Institut der Universität Bern, Sidlerstr. 5, 3012 Bern, Switzerland

5 Deutsches Zentrum für Luft- und Raumfahrt (DLR), Institut für Planetenforschung, Rutherfordstraße 2, 12489 Berlin, Germany

6 Centro di Ateneo di Studi ed Attivitá Spaziali "Giuseppe Colombo" (CISAS), University of Padova, via Venezia 15, 35131 Padova, Italy

7 LESIA-Observatoire de Paris, CNRS, Université Pierre et Marie Curie, Université Paris Diderot, 5 place J. Janssen, 92195 Meudon, France 
${ }^{8}$ Graduate Institute of Astronomy, National Central University, 300 Chung-Da Rd, 32054 Chung-Li, Taiwan

9 Department of Physics and Astronomy "G. Galilei", University of Padova, Vicolo dell'Osservatorio 3, 35122 Padova, Italy

10 Aix Marseille Université, CNRS, LAM (Laboratoire d'Astrophysique de Marseille) UMR 7326, 13388 Marseille, France

11 Centro de Astrobiología (CSIC-INTA), 28850 Torrejón de Ardoz, Madrid, Spain

12 International Space Science Institute, Hallerstrasse 6, 3012 Bern, Switzerland

13 Scientific Support Office, European Space Research and Technology Centre/ESA, Keplerlaan 1, Postbus 299, 2201 Noordwijk, The Netherlands

${ }^{14}$ PAS Space Research Center, Bartycka 18A, 00716 Warszawa, Poland

15 LATMOS, CNRS/UVSQ/IPSL, 11 Boulevard d'Alembert, 78280 Guyancourt, France
${ }^{16}$ INAF Osservatorio Astronomico di Padova, Vicolo dell'Osservatorio 5, 35122 Padova, Italy

17 CNR-IFN UOS Padova LUXOR, via Trasea 7, 35131 Padova, Italy

18 Department of Physics and Astronomy, Uppsala University, Box 516, 75120 Uppsala, Sweden

19 Department of Industrial Engineering, University of Padova, via Venezia 1, 35131 Padova, Italy

20 University of Trento, via Sommarive 9, 38123 Trento, Italy

21 INAF Osservatorio Astronomico di Trieste, via Tiepolo 11, 34014 Trieste, Italy

22 Instituto de Astrofísica de Andalucía-CSIC, Glorieta de la Astronomia, 18008 Granada, Spain

23 Operations Department, European Space Astronomy Centre/ESA, PO Box 78, 28691 Villanueva de la Cañada (Madrid), Spain

24 Department of Information Engineering, University of Padova, via Gradenigo 6/B, 35131 Padova, Italy 